\title{
Effects of diaphragm and deep abdominal muscle exercise on walking and balance ability in patients with hemiplegia due to stroke
}

\author{
Hye-Jin Lee', Tae-Woo Kang², Beom-Ryong Kim ${ }^{3, *}$ \\ 'Department of Social Physical Education, Wonkwang University, Iksan, Korea \\ ${ }^{2}$ Department of Physical Therapy, Wonkwang University School of Medicine \& Hospital, Iksan, Korea \\ ${ }^{3}$ Department of Physical Therapy, Design Hospital, Jeonju, Korea
}

The aim of this study was to investigate the effects of diaphragm and deep abdominal muscle exercise (DDAME) on walking ability (WA) and balance ability (BA) in subjects with hemiplegia due to stroke. Study subjects consisted of twenty patients diagnosed with hemiplegia due to stroke. They were randomized into a control group (CG) $(n=10)$ that performed traditional exercise and an experimental group (EG) $(n=10)$ that performed DDAME. The WA was measured using the 10-m walking test (10MWT) and 6-min walk test (6MWT). The BA was measured using the Timed Up and Go test (TUG) and Berg's balance scale (BBS). Paired $t$-test was performed to compare differences within the groups before and after intervention. The analysis of covariance was performed to compare the differences between the EG and CG. The level of significance was set at $\alpha=0.05$. Within-group changes in 10MWT and 6MWT were significantly different in both the EG $(P<0.05)$ and $C G$ $(P<0.05)$. There was significantly more improvement in the 10MWT and $6 \mathrm{MWT}$ in the EG than in the CG $(P<0.05)$. Within-group changes in TUG and $B B S$ were significantly different in both the $E G(P<0.05)$ and the $C G$ $(P<0.05)$. There was significantly more improvement in TUG and BBS in the EG than in the CG $(P<0.05)$. DDAME program, with diaphragm and inspiratory muscle strengthening exercises, is needed for patients experiencing difficulty in WA, BA, trunk asymmetry, abnormal alignment, mobility of trunk muscles, power, or endurance decline.

Keywords: Balance ability, Respiratory muscle exercise, Stroke, Walking ability

\section{INTRODUCTION}

Stroke is a major disease that can cause disability in adults and is the second leading cause of death in the world (Nilanont et al., 2014). In Korea, according to the cause of death statistics in 2014, cerebrovascular diseases are ranked third after cancer and heart disease. The single disease death rate is one of the most important causes of death in Korea (Statistics Korea, 2015).

Stroke dysfunction significantly degrades motor function due to muscle weakness, reduced motor control ability, and loss of coordinated movement on the affected side. This leads to a decrease in physical activities, such as independent walking ability (WA) and balance ability (BA), which decreases the efficiency of the

trunk stability muscles. These problems accelerate fatigue during a rehabilitation program or aerobic exercise that requires power and endurance. The functional recovery and rehabilitation community are delayed by bringing a limit to the WA and BA (Roffe et al., 2010).

Song and Kim (2010) showed that WA and BA improved after trunk stability exercise (TSE) was performed for 8 weeks by stroke patients. Choi et al. (2012) reported that the TSE and core stability exercises using gym-ball for elderly women for 4 weeks resulted in improved WA and BA. They emphasized the necessity of TSE for fall prevention.

Thus, the importance of abdominal muscles and paraspinal muscles in WA and BA is well known; however, the importance

${ }^{\star}$ Corresponding author: Beom-Ryong Kim (iD https://orcid.org/0000-0002-4592-4499 Department of Physical Therapy, Design Hospital, 390 Gyeonhwon-ro, Deokjin-gu, Jeonju 54910, Korea

Tel: +82-63-240-2520, Fax: +82-63-224-2275, E-mail: kimbr21@hanmail.net

Received: May 9, 2018 / Accepted: June 11, 2018 
of diaphragm as a trunk stability muscle has been overlooked. The diaphragm controls the intra-abdominal pressure by cocontraction with the abdominal muscles during breathing. In the inspiratory state, diaphragm contraction causes the air to enter the lung. During the peak expiratory phase, the diaphragm is relaxed in the superior direction to allow air to flow outside. Thus, the diaphragm and abdominal muscles control breathing by controlling intra-abdominal pressure (Talasz et al., 2011). In previous research related to the diaphragm, Sutbeyaz et al. (2010) showed an improvement in pulmonary function in stroke patients as a result of performing diaphragm exercise for 6 weeks. Patients' difficulty in breathing was reduced, resulting in improved quality of life. Britto et al. (2011) evaluated stroke patients who performed a diaphragm exercise with $30 \%$ resistance of maximal inspiratory mouth pressure for 8 weeks; the maximal inspiratory mouth pressure and muscle power and endurance of the inspiratory muscle were increased. Finally, Jung et al. (2014) performed electrical stimulation of the abdominal muscles simultaneously with diaphragm exercise over 4 weeks in stroke patients, which resulted in improved pulmonary function.

Thus, research on diaphragm function is underway, but previous studies only focused on improving the respiratory function. It is also necessary to study the effect of the diaphragm on abdominal muscles as well as on WA and BA. Therefore, this study investigates the changes in WA and $\mathrm{BA}$ in subjects with hemiplegia due to stroke after diaphragm and deep abdominal muscle exercise (DDAME) was performed for 6 weeks to investigate the effect of DDAME on WA and BA. Finally, the purpose of the study is to improve the functional symptoms of stroke patients and provide an effective exercise method.

\section{MATERIALS AND METHODS}

\section{Participants}

This study was conducted on patients in Jeonju city, Design Hospital for 6 weeks from March to April 2017. Subjects were diagnosed with hemiplegia due to stroke, between 50-70 years of age, diagnosed between 1 and 2 years previously, could perform 10-m walking independently and walk within 5-60 sec, Korean version of Mini- Mental State Examination (MMS E-K) score of 24 or more in order to understand and follow the indication of the researcher. Twenty subjects meeting inclusion criteria provided informed consented, and were explained the purpose and procedure for this study. They were randomized into a control group $(\mathrm{CG})(\mathrm{n}=10)$ with traditional exercise and experimental group
Table 1. General characteristics of subjects $(n=20)$

\begin{tabular}{lccc} 
Characteristic & $\begin{array}{c}\text { Experimental } \\
\text { group }(n=10)\end{array}$ & $\begin{array}{c}\text { Control group } \\
(\mathrm{n}=10)\end{array}$ & $P$-value \\
\hline Sex, male:female & $5: 5$ & $5: 5$ & 1.00 \\
Age $(\mathrm{yr})$ & $59.80 \pm 6.92$ & $60.20 \pm 8.24$ & 0.17 \\
Height $(\mathrm{cm})$ & $162.00 \pm 9.60$ & $161.30 \pm 8.77$ & 0.86 \\
Weight $(\mathrm{kg})$ & $63.30 \pm 7.44$ & $62.30 \pm 9.02$ & 0.50 \\
Side of stroke lesion, left:right & $7: 3$ & $6: 4$ & 1.00 \\
Stroke type, hemorrhage:infarction & $9: 1$ & $9: 1$ & 1.00 \\
Time since stroke (mo) & $17.20 \pm 4.37$ & $17.90 \pm 3.51$ & 0.91 \\
MMSE-K (score) & $26.50 \pm 2.22$ & $26.20 \pm 1.99$ & 0.27 \\
\hline
\end{tabular}

Values are presented as mean \pm standard deviation.

MMSE-K, Korean version of Mini-Mental State Examination.

\section{(EG) $(\mathrm{n}=10)$ with DDAME.}

The characteristics of the participants are summarized in Table 1.

\section{Traditional exercise}

Traditional exercise was used for 6 weeks, 5 days per week, once for $40 \mathrm{~min}$. Intervention was performed controlling intensities for warm-up, cool-down, mat activity, neurodevelopmental treatment (NDT), and gait training at patient level. The CG focused on TSE to enhance trunk control ability and included a stretching exercise for trunk flexibility.

\section{Diaphragm and deep abdominal muscle exercise}

DDAME consisted 20 min of inspiratory muscle exercise (IME) and 20 min of bracing exercise as reported in Grenier and McGill (2007), for 6 weeks and 5 days per week. The IME used POWERbreathe (POWERbreath International Ltd., Warwickshire, UK) equipment to strengthen the inspiratory muscle. Equipment was introduced in a standing position where the tongs were tied to the nose and the mouthpiece was held in the mouth. During breathing, the equipment provides expiratory resistance, but when inspiratory, the inspiratory muscle receives resistance to the inspiratory muscle due to the intensity control spring in the grip section. IME using this equipment intervened with Romer et al. (2002) protocol. The intensity of the IME measured maximal inspiratory mouth pressure (PImax) by subjects. Subject was to perform $50 \%$ of PImax 7 times per week, 10 times over 2 to 3 weeks, 15 times for 4 to 5 weeks, and 20 times over 6 weeks (Kilding et al., 2010).

In Grenier and McGill (2007), bracing exercise was performed by placing a pressure biofeedback (STABILIZER, Pressure Biofeedback Unit, DJO Global, St., Vista, CA, USA) device on the lumbar region of the subject and holding the pressometer at $40 \mathrm{mmHg}$. 
The pressure of the pressometer was raised to $70 \mathrm{mmHg}$ to allow all muscles to contract on the abdominal (Grenier and McGill, 2007). When the subject put pressure on the abdominal by himself, the researcher gave feedback on whether exercise was performed correctly. During the 6-week intervention, we checked motor control and gradually increased the holding time to $7,10,15$, and 20 sec.

\section{Measurement of WA}

The $10-\mathrm{m}$ walking test (10MWT) is a test method to measure WA. The 10MWT sets a total distance of $14 \mathrm{~m}$, marked at the beginning and end $2 \mathrm{~m}$ with tape, and the subject makes a 14-m distance from the sign "start." It is a method to measure time for $10-\mathrm{m}$ distance except beginning and end $2 \mathrm{~m}$ for acceleration and deceleration. The average time was recorded by repeated measurement 3 times (Dean et al., 2000; Walker et al., 2000). This test tool is a highly reliable tool with intrarater and interrater reliability $r=0.95-0.96$ (van Loo et al., 2004).

The 6-min walk test (6MWT) is a test tool for measuring walking endurance (Swisher and Goldfarb, 1998). It is used to test the walking endurance of various neurological and cardiopulmonary injury patients (Kosak and Smith, 2005). Recently, it has been used to test cardiopulmonary capacity and WA of chronic disease patients (Franceschini et al., 2009). The measurement method requires the subject to walk the greatest distance possible over 6 min. The tester measured the total distance walked to give the elapsed time every minute for the objective test. This test tool is a highly reliable tool with intrarater and interrater reliability $r=$ 0.97-0.99 (Eng et al., 2004).

\section{Measurement of BA}

Timed Up and Go test (TUG) is a test tool that can measure

Table 2. The comparison of variable on pre and post in group

\begin{tabular}{llcccl}
\hline Variable & \multicolumn{1}{c}{ Group } & Pre & Post & $t$ & $P$-value \\
\hline 10MWT (sec) & Experimental & $14.22 \pm 8.43$ & $12.93 \pm 7.93$ & 6.67 & $0.00^{* *}$ \\
& Control & $15.52 \pm 6.29$ & $14.81 \pm 5.89$ & 2.74 & $0.02^{*}$ \\
6MWT (m) & Experimental & $269.50 \pm 138.27$ & $294.00 \pm 144.62$ & -4.50 & $0.00^{* *}$ \\
& Control & $262.50 \pm 103.45$ & $272.00 \pm 110.11$ & -2.48 & $0.04^{*}$ \\
TUG (sec) & Experimental & $15.62 \pm 7.06$ & $12.93 \pm 6.23$ & 4.71 & $0.00^{* *}$ \\
& Control & $16.54 \pm 5.99$ & $16.20 \pm 5.89$ & 3.28 & $0.01^{*}$ \\
BBS (score) & Experimental & $41.80 \pm 8.95$ & $44.60 \pm 9.18$ & -5.71 & $0.00^{* *}$ \\
& Control & $40.40 \pm 10.94$ & $41.50 \pm 10.69$ & -3.16 & $0.01^{*}$ \\
\hline
\end{tabular}

Values are presented as mean \pm standard deviation.

10MWT, 10-m walking test; 6MWT, 6-min walk test; TUG, Timed Up and Go test; BBS, Berg's balance scale.

${ }^{*} P<0.05 .{ }^{* *} P<0.01$. mobility and balance quickly. The subject starts sitting comfortably on a 46-cm high armchair on a flat floor. It is a method of measuring the time required until one gets up from the chair and turns at the halfway point $3 \mathrm{~m}$ away from the chair, returns to the chair, and sits back in the chair. The average time was recorded by repeated measurement 3 times. This test tool is highly reliable tool with intrarater and interrater reliability $r=0.98-0.99$ (Podsiadlo and Richardson, 1991).

Berg's balance scale (BBS) is a test tool for objective test of static balance and dynamic BA. Fourteen items were applied to the three ambits of sitting, standing, and posture change from minimum 0 point to maximum 4 points, and the total score was 56 points. This test tool is a highly reliable tool with intra-rater and inter-rater reliability $r=0.97-0.99$ (Berg et al., 1995).

\section{Data analysis}

The statistical analysis was performed using IBM SPSS ver. 18.0 (IBM Co., Armonk, NY, USA). The Shapiro-Wilk test was used to check the normal distribution of data. The paired $t$-test was performed to compare differences in WA and BA within the groups before and after intervention. The analysis of covariance was performed to compare the differences between the EG and CG. The level of significance was set at $\alpha=0.05$.

\section{RESULTS}

\section{Comparison of WA changes}

Within-group changes in 10MWT were significantly different in both EG $(P<0.01)$ and CG $(P<0.05)$. Within-group changes in 6MWT were significantly different in both EG $(P<0.01)$ and

Table 3. The comparison of variable on between experimental and control groups

\begin{tabular}{llcccc}
\hline Variable & \multicolumn{1}{c}{ Group } & Pre & Post & $F$ & $P$-value \\
\hline 10MWT $(\mathrm{sec})$ & Experimental & $14.22 \pm 8.43$ & $12.93 \pm 7.93$ & 6.78 & $0.02^{*}$ \\
& Control & $15.52 \pm 6.29$ & $14.81 \pm 5.89$ & - & - \\
6MWT $(\mathrm{m})$ & Experimental & $269.50 \pm 138.27$ & $294.00 \pm 144.62$ & 5.36 & $0.03^{*}$ \\
& Control & $262.50 \pm 103.45$ & $272.00 \pm 110.11$ & - & - \\
TUG (sec) & Experimental & $15.62 \pm 7.06$ & $12.93 \pm 6.23$ & 21.02 & $0.00^{* *}$ \\
& Control & $16.54 \pm 5.99$ & $16.20 \pm 5.89$ & - & - \\
BBS (score) & Experimental & $41.80 \pm 8.95$ & $44.60 \pm 9.18$ & 7.73 & $0.01^{*}$ \\
& Control & $40.40 \pm 10.94$ & $41.50 \pm 10.69$ & - & - \\
\hline
\end{tabular}

Values are presented as mean \pm standard deviation.

10MWT, 10-m walking test; 6MWT, 6-min walk test; TUG, Timed Up and Go test; BBS, Berg's balance scale.

${ }^{*} P<0.05$. ${ }^{* *} P<0.01$ 
CG $(P<0.05)$ (Table 2$)$. There was significantly more improvement for 10MWT in EG than in CG $(P<0.05)$. There was significantly more improvement for $6 \mathrm{MWT}$ in $\mathrm{EG}$ than in $\mathrm{CG}$ $(P<0.05)$ (Table 3).

\section{Comparison of BA changes}

Within-group changes in TUG were significantly different in both EG $(P<0.01)$ and CG $(P<0.05)$. Within-group changes in BBS were significantly different in both EG $(P<0.01)$ and CG $(P<0.05)$ (Table 2$)$. There was significantly more improvement for TUG in EG than in CG $(P<0.01)$. There was significantly more improvement for BBS in EG than in CG $(P<0.05)$ (Table 3).

\section{DISCUSSION}

The purpose of this study was to investigate the effect of DDAME on WA (using 10MWT and 6MWT) and BA (using TUG and BBS) after DDAME was performed for 6 weeks by patients with stroke-induced hemiplegia.

The bracing exercise described by Grenier and McGill (2007) is an exercise method for stability and strengthening of the trunk, which increases the power and endurance by cocontraction between deep muscles and superficial muscles surrounding the trunk. Kang et al. (2015) reported that respiratory activity and BA were improved in hemiplegia (due to stroke) patients by performing bracing exercise for 6 weeks, and supported the exercise method appropriateness of this study. Moodie et al. (2011) applied 5 basic training principles—overload, progressive load, repetition, individualization, and specificity—so that resistance to diaphragm and inspiratory muscles can be effectively increased with increased power and endurance. In the present study, diaphragm and inspiratory muscles strengthening exercise was used to increase mobility, power, and endurance of trunk muscles weakened by hemiplegia based on the previous research.

Choi and Oh (2012) reported a significant improvement in within-group and between-group 10MWT with intensive 2-week chest mobility exercise. Kim and Shin (2013) demonstrated a significant improvement after intervention using 10MWT in the EG who utilized inspiratory and expiratory exercise equipment as subjects and hemiplegia patients who were diagnosed with strokes for more than 6 months, the between-group comparison was not significant. In this study, 10MWT significantly improved after the intervention in both EG with DDAME and CG. In the between-group comparison, there was a significant difference in the $\mathrm{EG}$, in which the DDAME was performed, compared with the
CG. These results indicate the increase in power and endurance of the abdominal deep muscle, and that the diaphragm and inspiratory muscles stabilize the trunk, thereby improving the overall coordination of the trunk and limbs, resulting in an increase in the stance phase of the affected side and swing phase of the unaffected side. The mat activity, NDT, and gait training in the CG and the EG also showed that the 10MWT improved after intervention.

Kim et al. (2015) reported that 40 patients with hemiplegia due to stroke were treated with the concept-based respiratory muscle exercises of proprioceptive neuromuscular facilitation in an EG. 6MWT was compared within-group and between-groups, and showed significant improvement. Jung et al. (2017) demonstrated significant improvement in stroke-induced hemiplegia patients in a comparison of within-group and between-group 6MWT when EG performed breathing exercises. In this study, 6MWT significantly improved after intervention in both EG with DDAME and CG. In the comparison between-groups, there was a significant difference in the EG, in which the DDAME was performed, compared with the CG. This result shows that DDAME stabilizes trunk in gait by increasing mobility, power and endurance of trunk muscles weakened by hemiplegia, and stability of trunk increases weight shifting ability and BA.

Song and Kim (2010) demonstrated a significant improvement in TUG as a result of TSE intervention for 8 weeks in 17 subjects with hemiplegia due to stroke. In a study by Choi et al. (2012), 24 patients aged 65 years or older were randomized into TSE and gym-ball exercise group. The groups performed their exercises for 6 weeks. The results showed significant improvement in TUG for both groups after 4 weeks and 6 weeks, and there was no significant difference between-groups. Kang et al. (2015) demonstrated significant improvement in 20 hemiplegia patients comparing within-group and between-group analysis of TUG where the EG performed abdominal muscle strengthening exercises. In this study, TUG was significantly improved before and after the intervention in both EG with DDAME and CG. In the comparison of between-groups, there was a significant difference in the EG in which the DDAME was performed compared to the CG. These results suggest that DDAME is a positive exercise method for improving TUG, and strengthening the diaphragm, inspiratory muscles, abdominal deep muscles, and superficial muscles needed to improve BA.

Shin et al. (2009) randomized 38 hemiplegia patients with stroke to 20 TSE and 18 traditional balance exercise groups. After 7 weeks of intervention, BBS was observed in both groups and 
showed significant improvement after intervention. Also, TSE was found more effective upon between-group comparison. Lee et al. (2012) randomized 30 hemiplegia patients to the TSE and trunk resistance exercise group and showed that BBS significantly improved after the intervention in both groups, and TSE was considered to be an appropriate exercise method for improving BA. Shim et al. (2014) randomized 15 subjects with hemiplegia due to stroke to an NDT and CG based on TSE and showed significant improvement in BBS in both groups, but the comparison between-groups showed significant difference. In this study, BBS was significantly improved before and after the intervention in both the EG and CG that performed DDAME. In the comparison between-groups, there was a significant difference in the EG in which DDAME was performed compared to the CG. These results suggest that the $\mathrm{BA}$ is improved by increasing the muscle power of the trunk directly. These findings agree with previous research; however, unlike the previous research, strengthening of diaphragm and inspiratory muscles may have affected abdominal pressure due to coadjustment of diaphragm and abdominal muscles, which may have affected BA. The inspiratory muscle is the diaphragm and the external intercostal muscle (Hawkes et al., 2007). The auxiliary inspiratory muscles are the sternocleidomastoid, scalene, trapezius (Pettersen, 2005), pectoralis major (Pettersen, 2006). Most of the muscles contributing to TS are thought to be more effective in BBS.

Thus, DDAME was found to be effective in improving WA and BA. Therefore, DDAME program with diaphragm and inspiratory muscles strengthening exercise inclusions is needed as some stroke patients experience difficulty in WA and BA with trunk asymmetry, abnormal alignment and mobility of trunk muscles, and declining power and endurance. This study was conducted in one medical institution; thus, it is difficult to generalize the study results for all stroke patients is limited. Additionally, the study subjects, number of doses, and daily life of the study subjects were not controlled.

\section{CONFLICT OF INTEREST}

No potential conflict of interest relevant to this article was reported.

\section{REFERENCES}

Berg K, Wood-Dauphinee S, Williams JI. The Balance Scale: reliability assessment with elderly residents and patients with an acute stroke.
Scand J Rehabil Med 1995;27:27-36.

Britto RR, Rezende NR, Marinho KC, Torres JL, Parreira VF, Teixeira-Salmela LF. Inspiratory muscular training in chronic stroke survivors: a randomized controlled trial. Arch Phys Med Rehabil 2011;92:184-190.

Choi SH, Lim JH, Cho HY, Kim IB, Kim MK, Lee HY. The effects of trunk stabilization exercise using swiss ball and core stabilization exercise on balance and gait in elderly women. J Korean Soc Phys Med 2012;7: 49-58.

Choi SJ, Oh DW. The effects of intensive chest mobility exercise on increasing pulmonary function and gait in stroke patients. Inst Spec Educ Rehabitation Sci 2012;51:221-239.

Dean CM, Richards CL, Malouin F. Task-related circuit training improves performance of locomotor tasks in chronic stroke: a randomized, controlled pilot trial. Arch Phys Med Rehabil 2000;81:409-417.

Eng JJ, Dawson AS, Chu KS. Submaximal exercise in persons with stroke: test-retest reliability and concurrent validity with maximal oxygen consumption. Arch Phys Med Rehabil 2004;85:113-118.

Franceschini M, Carda S, Agosti M, Antenucci R, Malgrati D, Cisari C; Gruppo Italiano Studio Allevio Carico Ictus. Walking after stroke: what does treadmill training with body weight support add to overground gait training in patients early after stroke?: a single-blind, randomized, controlled trial. Stroke 2009;40:3079-3085.

Grenier SG, McGill SM. Quantification of lumbar stability by using 2 different abdominal activation strategies. Arch Phys Med Rehabil 2007; 88:54-62.

Hawkes EZ, Nowicky AV, McConnell AK. Diaphragm and intercostal surface EMG and muscle performance after acute inspiratory muscle loading. Respir Physiol Neurobiol 2007;155:213-219.

Jung JH, Shim JM, Kwon HY, Kim HR, Kim BI. Effects of abdominal stimulation during inspiratory muscle training on respiratory function of chronic stroke patients. J Phys Ther Sci 2014;26:73-76.

Jung NJ, Na SS, Kim SK, Hwangbo G. The effect of the inspiratory muscle training on functional ability in stroke patients. J Phys Ther Sci 2017; 29:1954-1956.

Kang JI, Kim BR, Park SK, Yang DJ, Jeong DK, Kim JH. Effects of deep abdominal muscle strengthening exercises on pulmonary function and the ability to balance in stroke patients. J Korean Phys Ther 2015;27: 258-263.

Kilding AE, Brown S, McConnell AK. Inspiratory muscle training improves 100 and $200 \mathrm{~m}$ swimming performance. Eur J Appl Physiol 2010;108:505-511.

Kim CB, Shin JH, Choi JD. The effect of chest expansion resistance exercise in chronic stroke patients: a randomized controlled trial. J Phys Ther Sci 2015;27:451-453.

Kim JS, Shin WS. The effects of respiratory muscle strengthening training 
on pulmonary function and gait ability in subacute stroke patients. J Korean Soc Phys Med 2013;8:489-496.

Kosak M, Smith T. Comparison of the 2-, 6-, and 12-minute walk tests in patients with stroke. J Rehabil Res Dev 2005;42:103-107.

Lee JY, Park JS, Lee DH, Han SK, Roh HL. Effect of the trunk muscle stabilization training on balance for chronic stroke patients. J Korea Acad Soc 2012;13:1212-1219.

Moodie L, Reeve J, Elkins M. Inspiratory muscle training increases inspiratory muscle strength in patients weaning from mechanical ventilation: a systematic review. J Physiother 2011;57:213-221.

Nilanont Y, Nidhinandana S, Suwanwela NC, Hanchaiphiboolkul S, Pimpak T, Tatsanavivat P, Saposnik G, Poungvarin N; Thai Stroke Registry. Quality of acute ischemic stroke care in Thailand: a prospective multicenter countrywide cohort study. J Stroke Cerebrovasc Dis 2014; 23:213-219.

Pettersen V. Muscular patterns and activation levels of auxiliary breathing muscles and thorax movement in classical singing. Folia Phoniatr Logop 2005;57:255-277.

Pettersen V. Preliminary findings on the classical singer's use of the pectoralis major muscle. Folia Phoniatr Logop 2006;58:427-439.

Podsiadlo D, Richardson S. The timed "Up \& Go": a test of basic functional mobility for frail elderly persons. J Am Geriatr Soc 1991;39:142-148.

Roffe C, Sills S, Pountain SJ, Allen M. A randomized controlled trial of the effect of fixed-dose routine nocturnal oxygen supplementation on oxygen saturation in patients with acute stroke. J Stroke Cerebrovasc Dis 2010;19:29-35.

Romer LM, McConnell AK, Jones DA. Effects of inspiratory muscle training on time-trial performance in trained cyclists. J Sports Sci 2002;20:
547-562.

Shim HB, Cho HY, Choi WH. Effects of the trunk stabilization exercise on muscle activity in lumbar region and balance in the patients with hemiplegia. J Korean Soc Phys Ther 2014;26:33-40.

Shin WS, Kim CY, Lee DY, Lee SM. The effects of trunk stability exercise on dynamic balance in the persons with chronic stroke. J Korea Acad Soc 2009;10:2509-2515.

Song JM, Kim SM. The effect of trunk stability exercise on balance and gait in stroke patients. J Korean Soc Phys Med 2010;5:413-420.

Statistics Korea. Cause of death statistics effect in 2014. Daejeon (Korea): Statistics Korea; 2015.

Sutbeyaz ST, Koseoglu F, Inan L, Coskun O. Respiratory muscle training improves cardiopulmonary function and exercise tolerance in subjects with subacute stroke: a randomized controlled trial. Clin Rehabil 2010; 24:240-250.

Swisher AK, Goldfarb AH. Use of the six-minute walk/run test to predict peak oxygen consumption in older adults. Cardiopulm Phys Ther J 1998;9:3-5.

Talasz H, Kremser C, Kofler M, Kalchschmid E, Lechleitner M, Rudisch A. Phase-locked parallel movement of diaphragm and pelvic floor during breathing and coughing-a dynamic MRI investigation in healthy females. Int Urogynecol J 2011;22:61-68.

van Loo MA, Moseley AM, Bosman JM, de Bie RA, Hassett L. Test-re-test reliability of walking speed, step length and step width measurement after traumatic brain injury: a pilot study. Brain Inj 2004;18:1041-1048.

Walker C, Brouwer BJ, Culham EG. Use of visual feedback in retraining balance following acute stroke. Phys Ther 2000;80:886-895. 